\title{
EVALUATION OF PERSISTENT SCATTERER PATTERNS AT BUILDING FACADES BY SIMULATION TECHNIQUES
}

\author{
S. Auer ${ }^{\mathrm{a}}$, S. Gernhardt ${ }^{\mathrm{a}}$ and K. Eder ${ }^{\mathrm{b}}$ \\ ${ }^{\text {a }}$ Chair of Remote Sensing Technology, TU München, Arcisstr 21, 80333 München, Germany \\ $\{$ stefan.auer | stefan.gernhardt $\} @$ bv.tum.de \\ ${ }^{b}$ Dept. of Photogrammetry and Remote Sensing, TU München, Arcisstr 21, 80333 München, Germany \\ konrad.eder@bv.tum.de
}

KEY WORDS: Urban, high resolution, SAR, persistent scatterer, TerraSAR-X, simulation

\begin{abstract}
:
Thanks to the high resolution of modern SAR satellites many persistent scatterers (PS) appear at single buildings, approximately half of them representing building facades. These groups of points often show regular patterns in the SAR images that can be related to repeating structure elements on the facades. Hence, individual buildings can now be monitored over time, either based on amplitude (for change detection) and phase information (using interferometric methods). However, patterns of point signatures are often disrupted or disappear when the imaging geometry is slightly changed. The investigation at hand provides a detailed analysis of PS patterns based on reference data obtained from a photogrammetric survey. The processing of the optical images allows to create a detailed 3D model of the respective facade, which represents the geometrical object information for SAR simulation. From the simulation results the appearance of the point patterns of natural PS in high resolution SAR data can be better understood. The approach and findings for a case study in Munich is described in detail in this paper.
\end{abstract}

\section{MANUSCRIPT}

\subsection{Introduction}

The benefit of meter resolution SAR data of urban areas for Persistent Scatterer Interferometry (PSI) (Ferretti et al., 2001), (Adam et al., 2004), (Hooper, 2007) already has been demonstrated in the past years (Adam et al., 2008), (Gernhardt et al., 2010), (Gernhardt and Bamler, 2012). Due to the high resolution of up to approximately one meter and the side looking geometry of the SAR sensors, many persistent scatterers (PS) appear at building facades. These signatures very often show regular patterns because of repeating structures at the facade of modern buildings, like windows or balconies with metal or concrete borders. However, the analysis of PSI results from different orbital positions has revealed disappearing or disrupted PS patterns for specific urban scenes.

The origin of the point signatures and changes within the PS patterns due to varying acquisition geometries can only be interpreted and determined precisely by SAR simulation. To this end, $3 \mathrm{D}$ models of the buildings are required that include sufficient structural details. For the analysis at hand, a building facade is selected based on the appearance of unexpected phenomena - in the image data or in the PSI results - and is recorded by a digital photogrammetric camera. The goal of this procedure is the derivation of geometric object information (3D coordinates) of the facade. This data is used in a subsequent step for generating a detailed 3D model representing the geometry of facade structures such as balconies or window corners as well as the facade topology (structure regularities).

The digital images are first evaluated in a photogrammetric software environment (ERDAS imagine) wherein several coordinates of distinct facade features are extracted. Thereafter, this data and the additional measurements are used for creating a 3D model of each facade. In this regard, the focus is set on the detailed modeling of individual parts which regularly cover the facades. Afterward, the 3D models are imported and simulated in RaySAR, a SAR simulator developed at TUM (Auer et al., 2010). This software based on ray tracing techniques is capable of 3D SAR simulation with focus on the geometric correctness of simulated images and signal coordinates. The results include the 3D coordinates of signal phase centers (azimuth, range, elevation) and simulated images where different reflection levels are separable (single, double, triple bounce, etc.). Finally, the comparison of the simulated image with the real SAR image reveals the reason for unexpected phenomena within the PS patterns. In this context, the interpretation is further supported by linking the simulated 3D phase centers with the facade geometry.

The paper at hand is divided into two main sections. The first section in the following provides information on the photogrammetric survey, on the $3 \mathrm{D}$ modeling and on the simulation procedure, whereas the second one deals with the analysis of the simulation results in comparison to PS on real SAR image data.

\subsection{D Facade Model Generation}

1.2.1 Data Acquisition: The test site was selected based on the fulfillment of several prerequisites. The facade should include repeating structural elements in order to simplify the photogrammetric assessment and 3D modeling procedure. In this case one element can be modeled in detail and can be repetitively copied according to the respective position of recurrence measured from the images. In addition, a pattern of signatures should be present at the facade location in the SAR data and PS results, respectively, that disrupt/disappear with varying incidence angles. From recent studies PSI results of two descending tracks are available that are suitable for this investigation. The SAR data was acquired by the TerraSAR-X satellite in high resolution spotlight mode, from orbit 78 , beam 19 , and from orbit 2 , beam 50 , with (mid scene) incidence angles of $25.2^{\circ}$ and $39.4^{\circ}$, respectively. The acquisitions cover the central part of Munich, Germany, with an area of approximately $56 \mathrm{~km}^{2}$ and $20 \mathrm{~km}^{2}$. For the investigation at hand a suitable building to the west of central train station has been identified by visual inspection, labeled "Marsstrasse" in the following (by reason of its location at one end of this street).

For the 3D facade reconstruction a photogrammetric approach was used, with the advantage of very short time effort on the building site. In a first step, a local cartesian coordinate system 


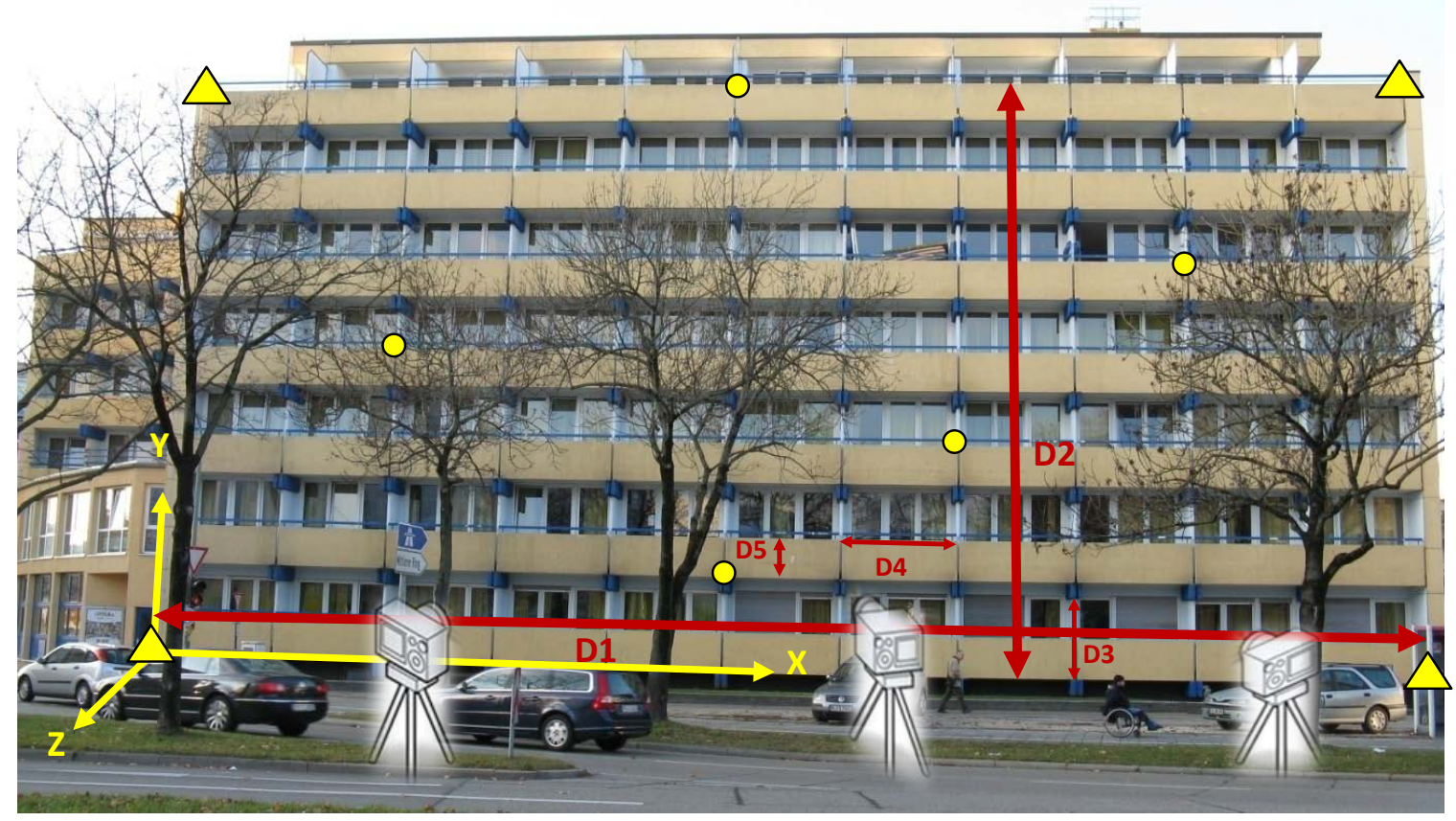

Figure 1: overview image of the facade with coordinate system, measured distances, control points (triangles: full CP, circles: height $\mathrm{CP}$ ) and camera directions (schematically). Additional measurements: total length of facade (D1), total height of facade (D2), distance between two balcony carriers (D3), width of balcony parapet wall (D4) and height of balcony parapet wall (D5).

was defined based on the assumption that the facade can be fitted to a main plane and the building primitives are rectangular to each other. Control points (CPs) are necessary for referencing the images (determination of the exterior orientation) within this system. In total 4 full control points ( $\mathrm{x}, \mathrm{y}$, and $\mathrm{z}$ coordinate is given) and 5 height control points (only $\mathrm{z}$ coordinate is given) have been determined by distance measurements with a laser range finder (Leica DISTO). Figure 1 shows an overview image of the facade with the local coordinate system, the distance measurements, and the control points. Furthermore, some small scale elements (like the width and depth of the metal balcony railing) are measured with a yard stick as they cannot be recorded during the photogrammetric assessment due to expected imprecision.

The images were taken with a NIKON D3 Camera which has been calibrated before. In order to avoid occlusions by cars and pedestrians, the images were shot from a stepladder about $3 \mathrm{~m}$ above ground level. Two types of images have been acquired:

- Stereoscopic overview images (distance to facade $30 \mathrm{~m}$ ) served as overall information source for the identification of control points, distance measurements, and the locations of repeating facade elements.

- Detail images (distance to facade about $12 \mathrm{~m}$, baseline about $10 \mathrm{~m}) .3$ images per station: perpendicular, tilted left, tilted right (about $50 \%$ overlap)

1.2.2 Photogrammetric Assessment: The photogrammetric processing comprised two steps in order to extract all necessary information for the 3D modeling.

Step 1: set up of a bundle block of the facade with a manual measurement of control and tie points (70 in total) within all relevant images (minimum 3 rays per point). The bundle block adjustment supplies the exterior orientation parameters of each image and the three dimensional coordinates of the tie points. These points served as anchor points for fitting the facade elements (cf. step 2). Table 1 shows the results of the bundle block adjustment.
The mean residuals at the control points are between $1.0 \mathrm{~cm}$ to $2.6 \mathrm{~cm}$.

Step 2: the perpendicular images served as stereoscopic models for stereo restitution with the software Stereo Analyst of ERDAS imagine. Within these stereo models 3D coordinates of all relevant features have been recorded and stored as 3D shape files. The accuracy (root mean square error, rsme) of the object points is below $3 \mathrm{~cm}$ in all directions (see Table 1). During data acquisition advantage was taken from the fact, that facade elements are repeating in a regular way. The coordinates of these elements have been recorded once including all relevant details. All (assumed) identical facade elements are created during the modeling by cloning this template at the respective positions. The resulting model is exported in DXF format for the adjacent 3D modeling procedure

\begin{tabular}{|l|l|l|l|l|}
\hline Facade & $\begin{array}{l}\text { no. of } \\
\text { images }\end{array}$ & $\begin{array}{l}\sigma_{0} \text { of } \\
\text { measured } \\
\text { image } \\
\text { coordinates }\end{array}$ & $\begin{array}{l}\text { mean } \\
\text { residual } \bar{v} \\
\text { at control } \\
\text { points }[\mathrm{m}]\end{array}$ & $\begin{array}{l}\text { rmse at } \\
\text { object } \\
\text { points } \\
{[\mathrm{m}]}\end{array}$ \\
\hline \hline $\begin{array}{l}\text { Mars- } \\
\text { strasse }\end{array}$ & 9 & 12.6 mikrons & $\begin{array}{l}\bar{v}_{x}=0.026 \\
\bar{v}_{y}=0.010\end{array}$ & $\begin{array}{l}\mathrm{x}: 0.028 \\
\mathrm{y}: 0.024 \\
\bar{v}_{z}=0.015\end{array}$ \\
z: 0.025
\end{tabular}

Table 1: Results of the bundle block adjustment for the facade "Marsstrasse".

1.2.3 3D Modelling: The 3D model of the facade is created in Sketchup, a 3D modeling software of Trimble (formerly distributed by Google). This software facilitates a straightforward construction of buildings with a manageable amount of tools and basic shapes. Especially the rectangular shapes and linear structures at the building at hand accelerate the modeling procedure, as the software is designed for objects assembled from these basic elements.

At the beginning the 3D coordinates of the photogrammetric as- 


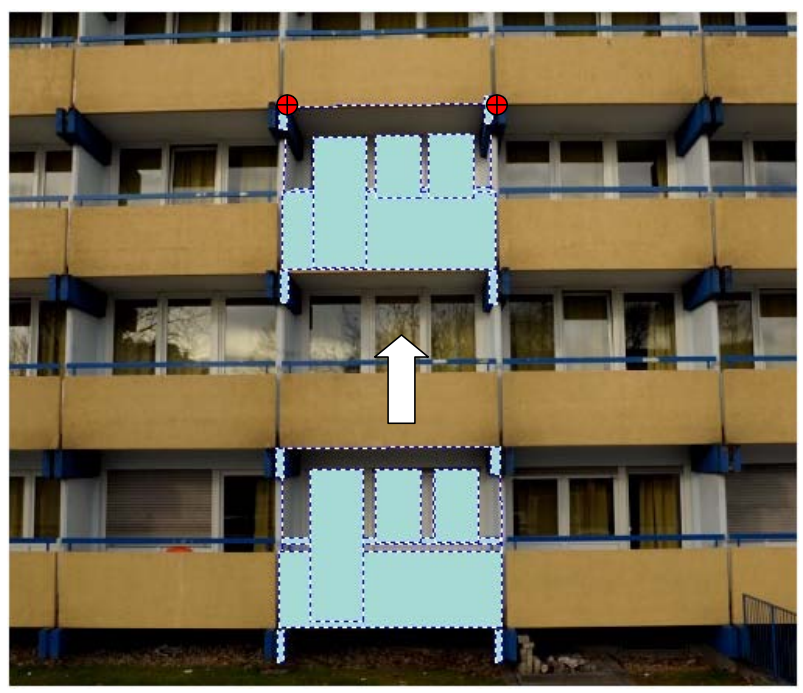

Figure 2: Facade mapping taking benefit of repeating features, fitted with anchor points.

sessment are imported into the software. The recorded points are used to model one facade element, special particular structural elements (e.g., the uppermost floor and roof) and the basic shape of the facade. During this step additional measurements of some object details are used that have been acquired on site, as mentioned in section 1.2.1. The structural details of the facade element are created using the basic tools of push/pull planes, parallel offset of polygons and the translation functionality. The completed element is duplicated at all appropriate positions, whereas the anchor points are used for precise positioning. Figure 2 shows the basic idea how a facade pattern is copied and fitted to another floor during modeling based on anchor points. Finally, all planes corresponding to glass panels are grouped in one layer in order to be able to differentiate metal/concrete from glass in the subsequent simulation.

The result of the 3D modeling is shown in Figure 3. From the detailed view the incorporated details become evident, i.e., the concrete bearing sticking out (in perpendicular) of the facade, the balcony railing and the peculiarities of the balcony door and windows with intermediate elements. The orientation and location of the facade in the world coordinate system (WGS84) is derived from reference data provided by Landesamt für Vermessung und Geoinformation, Munich. The overall accuracy of the model can be assumed to be in the order of $2-3 \mathrm{~cm}$ in all 3 coordinate axes, due to the limited accuracy of the object points measured from the stereo restitution. However, this precision is sufficient for the investigation at hand and within expectation for facade reconstructions using the presented methodology. Further improvements can only be achieved either by different measurement techniques (more time consuming) or incorporating, e.g., a UAV with a camera in order to avoid oblique viewing especially at the upper parts of the facade.

1.2.4 SAR Simulation: The simulation part is conducted using RaySAR (Auer et al., 2010) (Auer, 2011), a 3D SAR simulator based on an enhancement of the open-source ray tracer Pov-RAY. The preface 3D relates to the simulation of radar signals in azimuth, range, and elevation. The concept of RaySAR corresponds to the implicit assumption of standard SAR processing that the direct backscattering of signals is dominant (Born approximation). Accordingly, the simulation results can be used as a reference which is comparable with real SAR images (appearance and position of signatures in azimuth-range plane) and results from interferometric algorithms (location of signal phase

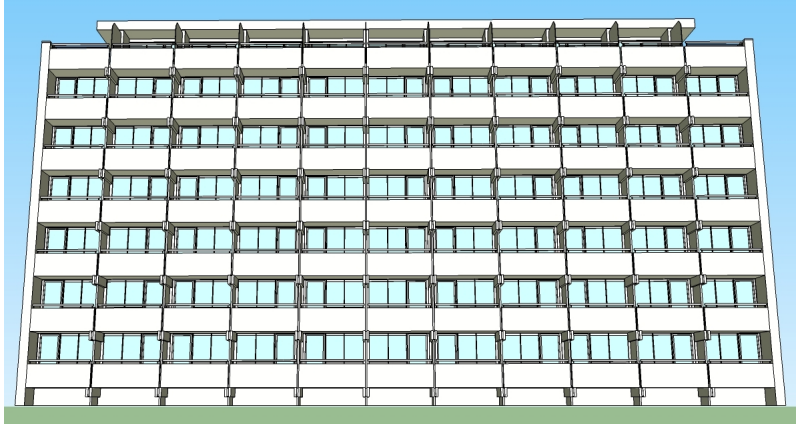

(a) 3D Model of facade

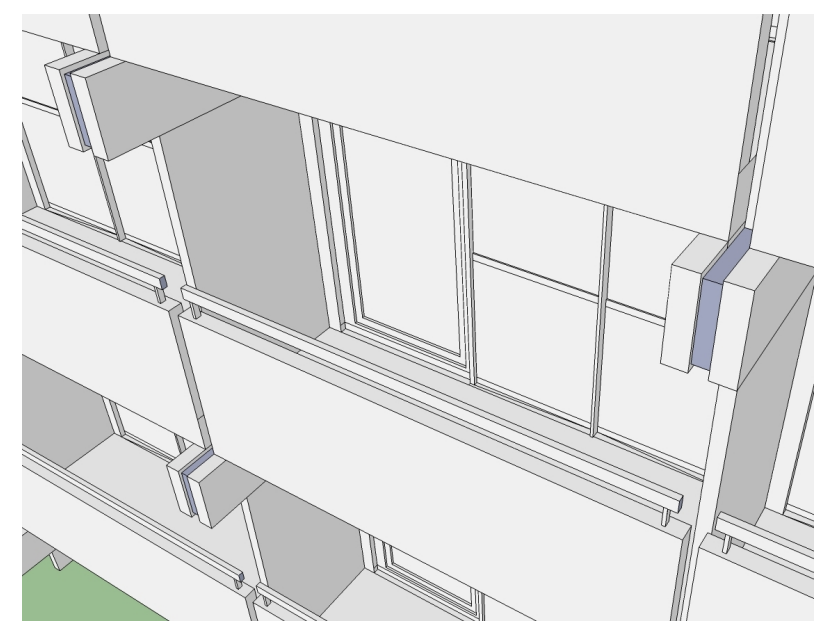

(b) Detailed view on part of facade model

Figure 3: Overview on the 3D model of the facade located at Marsstrasse (top). Several constructional details are included that can be seen from a slight side view (bottom).

centers in $3 \mathrm{D})$. The basic steps of the simulation procedure are detailed below.

As a first step, the building geometry, originally defined in CAD software, is transferred to the POV-Ray environment. Therein, the geometric and radiometric scene settings are adapted to SAR imaging. The geometric part considers the following parameters: signal incidence angle, building orientation with respect to the sensor's line-of-sight, and pixel spacing. The signal incidence angle is assumed to be locally constant as the building facade is located in the far field of the radar antenna (flat wave front). Following this assumption, the simulation of signals is directly conducted in three dimensions without raw data processing. When generating the SAR image, the pixel spacing in azimuth and range is adapted to the real SAR case, i.e., TerraSAR-X high resolution spotlight mode as shown in the case study below. The building geometry remains unchanged as the facade model is defined in absolute coordinates. Finally, the number of rays in the azimuthelevation plane is defined, representing a discrete form of the signal wave front. For the case study at hand, the density is adapted to the size of relevant corners, e.g., smaller than $5 \mathrm{~cm}$.

The radiometric part considers different aspects. The model for signal reflections is an approximate solution, adapted to the appearance of reflections of Fresnel type (Knott et al., 2004) and diffuse reflections from surfaces with little roughness (small perturbation method), e.g., (Franceschetti et al., 2001). For the facade model at hand, the surfaces are characterized by little/negligible roughness and material made of metal or glass. Facade surfaces are assigned with stronger reflectance than ground areas. The signal penetration of glass is represented by $100 \%$ signal absorption. 


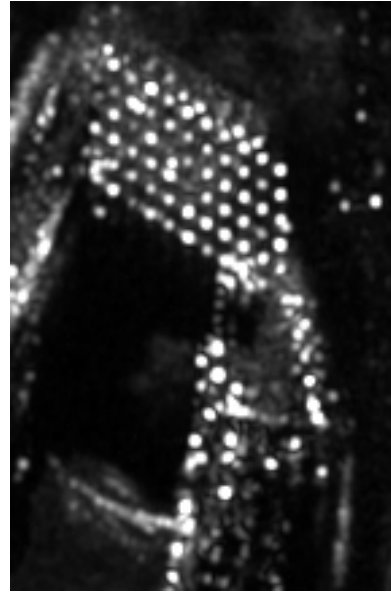

(a) Temporal average image, incidence angle: $39.09^{\circ}$

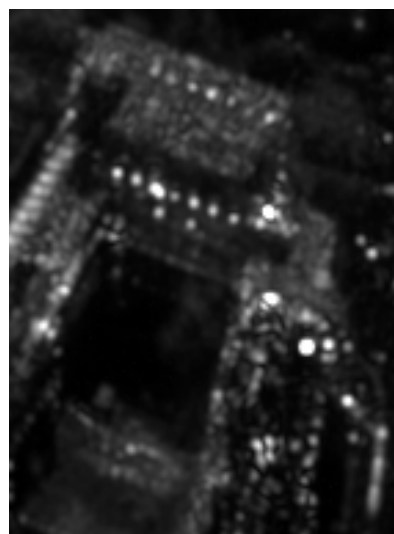

(e) Temporal average image, incidence angle: $24.91^{\circ}$

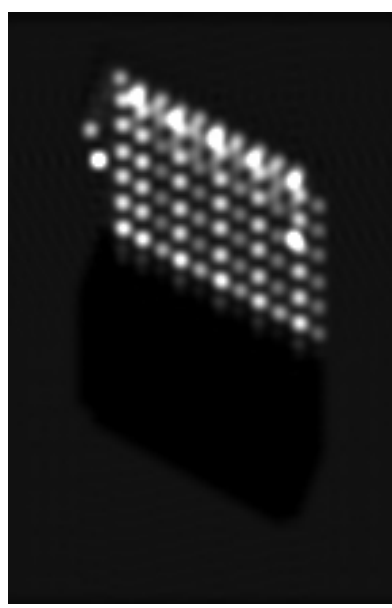

(b) Simulated image

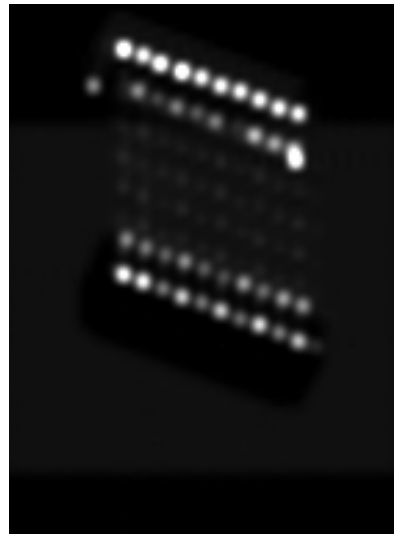

(f) Simulated image

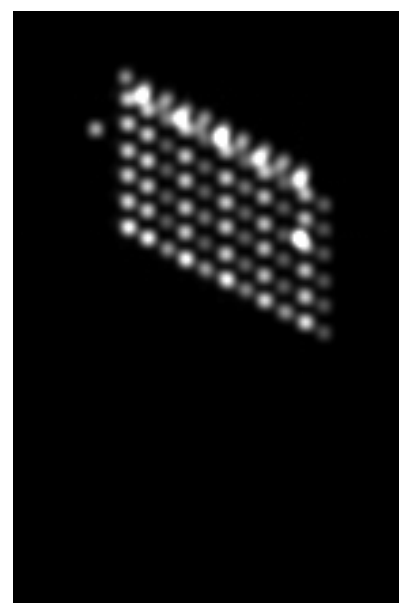

(c) Triple reflection

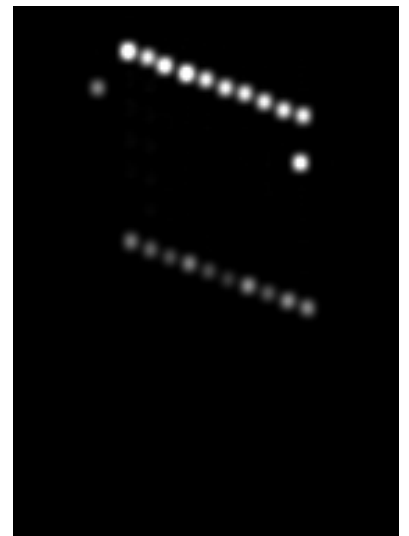

(g) Triple reflection

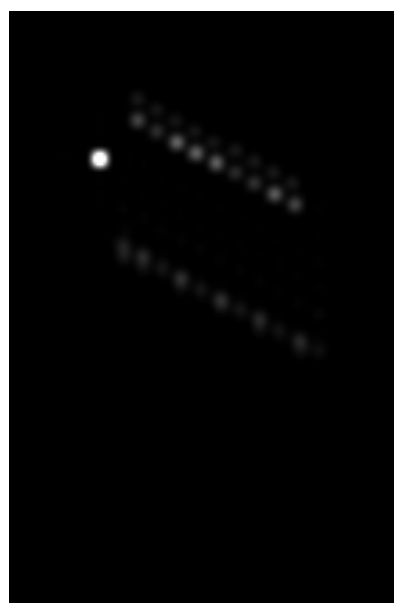

(d) Fivefold reflection

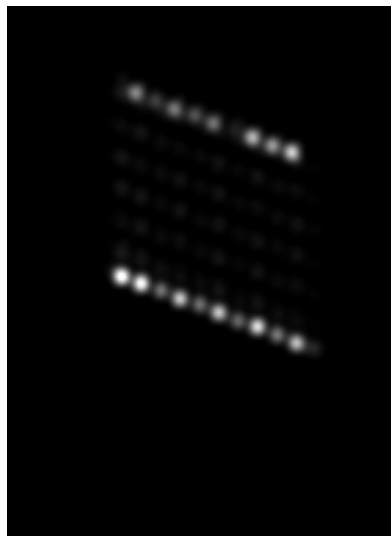

(h) Fivefold reflection

Figure 4: Comparison of TerraSAR-X temporal average images with simulated images and relevant image layers related to different reflection levels. Azimuth: left to right. Range: top-down.

That is, for the sake of simplicity signals entering the building are not expected to return. For creating the SAR image, signal contributions are summed coherently within the resolution cells. The image resolution is adapted to TerraSAR-X high resolution spotlight mode (impulse response imposed by hamming window for suppressing signal sidelobes).

Based on the geometric and radiometric parameters, the following simulation results are provided using RaySAR:

- Images in SAR geometry: Besides a reflectivity map marking the distribution of signals in the azimuth-range plane, a SAR image is simulated for direct comparison with the real SAR image. Moreover, separate image layers are generated for different reflection levels, e.g., triple and fivefold bounce.

- 3D coordinates of simulated signal phase centers: The discrete positions of signal contributions are written to a CAD file representing signal phase centers. Focusing on specular reflections - marked by a flag in RaySAR during the ray tracing procedure - the positions of dominant signals can be analyzed in detail, for instance, by mapping them into the detailed facade model.

\subsection{Facade Analysis}

As introduced in Section 1.2.1, the simulation case study "Marsstrasse" is related to the unexpected disappearing of PS candidates for different signal incidence angles. In order to explain this phenomenon, the simulation results are analyzed in detail in this section. In this context, simulated images are compared to the real SAR image (analysis in 2D). Moreover, the simulated phase centers are mapped into the facade model in order to check the correspondence between the localized signal and the facade structure.

1.3.1 Analysis in 2D: As the focus is on deterministic image components, it is reasonable to use temporal average images of the given SAR data stacks for comparison, where the speckle effect is suppressed significantly without loss of resolution. Figures $4 \mathrm{e}$ and $4 \mathrm{a}$ show the temporal average images of the urban scene "Marsstrasse" for incidence angles of $24.91^{\circ}$ and $39.09^{\circ}$ (TerraSAR-X high resolution spotlight mode, ascending orbit, 8 bit images with clipped intensity). Obviously, the dominant pattern of signatures only occurs for the bigger incidence angle whereas the building appears to be almost invisible for the smaller angle. In both images, the underlying patch of medium gray level is related to diffuse signal response from the flat roof surface.

The simulated images support the visual interpretation of dominant signatures in the TerraSAR-X image. As specular reflections are activated at all building surfaces, the simulation provides an 


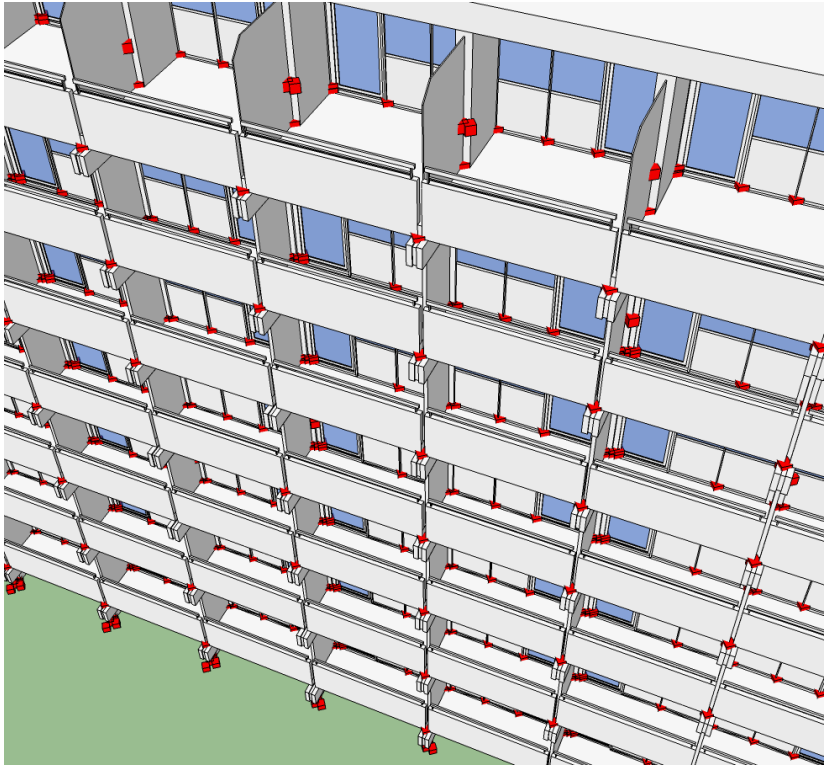

(a) Simulated phase centers for signal incidence angle of $39.09^{\circ}$. Corner reflections appear in the balcony interior (dominant) and on the balcony railing (negligible).

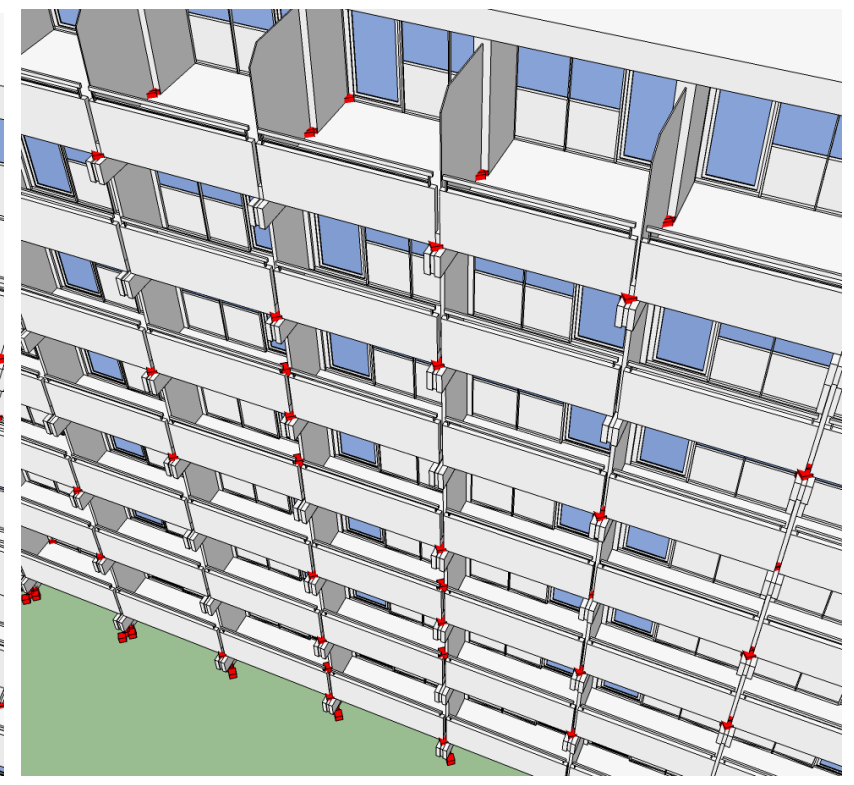

(b) Simulated phase centers for signal incidence angle of $24.91^{\circ}$. Corner reflections in the balcony interior are disabled with except for the uppermost floor.

Figure 5: Simulated phase centers related to reflection level 3 marked in facade model (red cubes). The change of the signal incidence angle leads to a loss of corner reflections at the balcony interior.

ideal result in the azimuth-range plane where the appearance of signatures is mainly related to the building and imaging geometry. Accordingly, it is possible to look out for the expected signature positions in the real SAR images and to explain effects related to the variation of the signal incidence angle. In that regard, the analysis can be further concentrated on specific image signatures, as separate image layers are simulated for different reflection levels. As a general remark, the simulated images reveal for both signal incidence angles that the dominant signal contributions of the scene "Marsstrasse" are related to reflection levels 3 and 5. In contrast, reflection levels 1,2, and 4 are negligible as the strength of the respective signals is close to zero. A detailed comparison with the real SAR images is given next.

In case of an incidence angle of $39.09^{\circ}$, triple reflections dominate whereas fivefold reflections are expected to be weak (see Fig. 4b-4d). This is mainly related to corners formed by the facade and the balcony structure. Hence, the simulation gives strong hints that the dominant signature pattern in the TerraSAR$\mathrm{X}$ image, selected as persistent scatterers in the stack, are apparently related to reflection level 3. In contrast, fivefold reflections are negligible or not present in the SAR image. For an signal incidence angle of $24.91^{\circ}$ (see Fig. 4e), the building is only represented by two dominant signature rows in the TerraSAR-X image and areas of low gray value. The upper row of dominant point signatures is confirmed by the simulation and is related to triple reflection (see dominant signature row in Fig. 4g). In contrast, the lower row of signatures is related to corners on the rear part of the building roof which are not represented by the building model. Accordingly, the signature row does not occur in the simulated image. The invisibility of the facade is confirmed by the simulated image. The reason is that the corners in the balcony interior are hidden by the balcony railing and, hence, are not in the line-of-sight of the sensor. Instead, radar signals have to follow five specular reflections at the balcony structure in order to return to the SAR sensor (see simulated layer in Fig. 4h). To this end, all balcony surfaces have to be characterized by strong reflectance avoiding the loss of signal strength, what is obviously not the case for the "Marstrasse" building. Finally, the simula- tion reveals signature rows located at the lower end of the facade layover (triple reflections) and in the shadow area (fivefold reflections). Both effects are not present in the real SAR image as the ground next to the facade is mainly covered by grass, where specular reflections are disabled.

As a conclusion, the simulation case study "Marsstrasse" gives an example where the constellation of balcony geometry and sensor's line-of-sight is responsible for the disappearance of persistent scatterers. Moreover, the roughness of the ground below the building deactivates a high number of expected reflections. Besides the interest in the context of interferometric methods, the results of the case study are also interesting for change detection applications. Facades may fully disappear in case of a change of the incidence angle while signature rows in the building layover may be mistakenly interpreted as facade structures.

1.3.2 Simulated 3D phase centers: The simulated image layers indicate that the dominant signal contributions in both SAR images are related to triple reflections. As RaySAR also covers the elevation direction, the simulated phase centers can be also compared with the results of interferometric algorithms. Hence, as a next step in the course of the "Marsstrasse" case study, the correspondence of the signal phase centers and the facade geometry is checked visually. To this end, the phase centers are projected into the building model based on the known imaging geometry (sensor position, signal incidence angle, aspect angle with respect to the facade) and the 3D coordinates (azimuth, range, elevation). The merged scenes are visualized in Figure 5 where triple reflections of specular type are represented by red cubes. In case of an incidence angle of $39.09^{\circ}$, the signal phase centers are mostly located at corners in the interior of balconies. In this context, only corners with larger scale yield dominant signals in the simulated SAR image. The regularity of those balcony corners leads to the regular signature pattern in the simulated SAR image (see Fig. 4c). In case of the lower incidence angle, the uppermost building floor is represented by only one row of phase centers. A high number of triple reflections is lost as the corners of large scale in the balcony interior are not in the line-of-sight of the SAR sensor. Some triple reflections on the balcony fronts remain but 
do not contribute significantly to the simulated SAR image due to low amplitude. Triple reflections on the ground level (red cubes on green surface) are negligible for both signal incidence angles due to the ground roughness (covered by grass).

\subsection{Conclusion}

In the context of understanding the nature of persistent scatterers, a case study has been conducted which fulfills two aims: A.) explaining the unexpected disappearance of PS in case of different signal incidence angles and B.) providing a reference for evaluating the localization capability of PSI for high resolution TerraSAR-X data stacks. To this end, a very detailed facade model has been created based on photogrammetric survey and has been simulated using RaySAR, a software based on ray tracing techniques. The simulation reveals that the unexpected invisibility of a selected facade is related to a specific constellation between the facade and imaging geometry. Moreover, simulated 3D phase centers have been related to physical balcony corners of the facade model. Future work will be concerned with comparing the position of localized, geocoded PS with the simulated reference.

\subsection{Acknowledgement}

The work presented in the paper is partly funded by the Deutsche Forschungsgemeinschaft (DFG), project VHR-SAR (BA 2033/3$1)$.

\section{REFERENCES}

Adam, N., Eineder, M., Yague-Martinez, N. and Bamler, R., 2008. High resolution interferometric stacking with TerraSAR$X$. In: IEEE Transactions on Geoscience and Remote Sensing, Vol. 2, pp. 117-120.

Adam, N., Kampes, B. and Eineder, M., 2004. Developement of a scientific permanent scatterer system: Modifications for mixed ERS/ENVISAT time series. In: In: Proceedings of Envisat \& ERS Symposium, Salzburg, Austria, on CD-ROM.

Auer, S., 2011. 3D Synthetic Aperture Radar Simulation for Interpreting Complex Urban Reflection Scenarios. PhD thesis, Deutsche Geodtische Kommission, Reihe C, Nr. 660, Verlag der Bayerischen Akademie der Wissenschaften, ISBN 978-3-76965072-3, 126 p., http://dgk.badw.de/fileadmin/docs/c-660.pdf.

Auer, S., Hinz, S. and Bamler, R., 2010. Ray-tracing simulation techniques for understanding high-resolution SAR images. 48(3), pp. 1445-1456.

Ferretti, A., Prati, C. and Rocca, F., 2001. Permanent scatterers in SAR interferometry. 39(1), pp. 8-20.

Franceschetti, G., Iodice, A. and Riccio, D., 2001. Fractal Models for Scattering from Natural Surfaces. Academic Press, chapter 1.6.5, pp. 467-485.

Gernhardt, S., Adam, N., Eineder, M. and Bamler, R., 2010. Potential of very high resolution SAR for persistent scatterer interferometry in urban areas. Annals of GIS 16(2), pp. 103-111.

Gernhardt, S. and Bamler, R., 2012. Deformation monitoring of single buildings using meter-resolution sar data in psi. ISPRS Journal of Photogrammetry and Remote Sensing 73(0), pp. 6879 .
Hooper, A., 2007. A combined multi-temporal InSAR method incorporationg persistent scatterer and small baseline approaches. In: In: Proceedings of FRINGE Workshop, Frascati, Italy, pp. on CD-ROM.

Knott, E., Shaeffer, J. and Tuley, M., 2004. Radar Cross Section. 2nd edition edn, SciTech Publishing, NC. 